SU-ITP-05/26

SLAC-PUB-11510

hep-th/0510044

October 2005

\title{
Dimensional Mutation and Spacelike Singularities
}

\author{
Eva Silverstein \\ SLAC and Department of Physics, Stanford University, Stanford, CA 94305-4060
}

I argue that critical string theory on a Riemann surface of genus $h>>1$ crosses over, when the surface approaches the string scale in size, to a background of supercritical string theory with effective central charge as large as $2 h$. Concrete evidence for this proposal is provided by the high energy density of states (realized on the Riemann surface side by strings wrapping nontrivial elements of the fundamental group) and by a linear sigma model which at large $h$ approximates the time evolution through the initial transition. This suggests that cosmological singularities arising in negatively curved FRW backgrounds may be replaced by a phase of supercritical string theory. 


\section{Dimensionality and its Discontents}

Like spacetime itself, the notion of dimensionality is a derived concept. In the context of perturbative string theory, a more precise diagnostic for the effective dimensionality is the effective matter central charge $c_{e f f}$, which determines the high energy density of states of the system. In simple situations, such as flat space or linear dilaton backgrounds, this corresponds to the number of dimensions in which strings can oscillate, but it applies equally well to small target spaces where the geometrical description breaks down. In appropriate circumstances [1], the worldsheet modular group relates $c_{e f f}$ to the Zamalodchikov $c$ function appropriate to the worldsheet matter sector.

For example, in noncritical limits of string theory, the worldsheet Weyl anomaly conditions are equivalent to the equations of motion following from a spacetime effective action with a string-frame effective potential [2]

$$
\mathcal{V}_{d}^{(s)} \sim \frac{1}{g_{e f f}^{2}} \frac{D-D_{c}}{l_{s}^{d}}
$$

Here $D_{c}$ is the critical dimension, $g_{s}$ is the string coupling, and $g_{e f f}^{2}=g_{s}^{2} / V$ for a compactification from $D$ to $d$ dimensions on a space of volume $V l_{s}^{D-d}$. That is, the Weyl anomaly has a contribution proportional to the central charge $D-D_{c}$ of the matter sector, which must be balanced against other contributions (such as time dependence in the dilaton). Correspondingly, the string can independently oscillate in $D$ directions (modulo the ghost contributions which effectively remove two dimensions) and the high energy density of states grows exponentially with the mass times $\sqrt{D}$.

Now consider critical string theory on a target space which includes a genus $h$ Riemann surface component $\Sigma$ of volume $V_{\Sigma} l_{s}^{2}$. Dimensional reduction to eight dimensions on a constant curvature surface yields a string frame effective potential

$$
\mathcal{V}_{8}^{(s)} \sim \frac{1}{g_{e f f}^{2}} \frac{2 h-2}{V_{\Sigma} l_{s}^{8}}
$$

Comparing (1.1)(1.2), we see that the Weyl anomaly for $g_{\text {eff }}$ scales like $D-D_{c}$ in the supercritical case and like $(2 h-2) / V_{\Sigma}$ in the Riemann surface case. This suggests an effective central charge

$$
c_{\text {eff }}^{W e y l} \sim \frac{2 h-2}{V_{\Sigma}}
$$

At large volume $V_{\Sigma} \rightarrow \infty$, the theory reverts to ten dimensional flat space, for which the central charge (or $\hat{c}$ in the superstring) of the Riemann surface direction is 2 . This 
corresponds to the number of dimensions in which the string can oscillate. As $V_{\Sigma}$ decreases, the effective central charge appearing in the Weyl anomaly increases. As the volume $V_{\Sigma} l_{s}^{2}$ approaches the string scale, this effective central charge approaches $2 h-2$. More precisely, when $V_{\Sigma}$ is of order $2 h-2$, the curvature $\mathcal{R} \sim(2 h-2) /\left(V_{\Sigma} l_{s}^{2}\right)$ reaches the string scale, and the formulas $(1.2)(1.3)$ can be significantly corrected. In any case, $c_{\text {eff }}^{\text {Weyl }}$ increases as the volume of the surface decreases, suggesting a connection between negatively curved target spaces at small radius and supercritical strings.

Now, this increase in the central charge for smaller size might at first sight appear surprising, as it does not correspond to the naive number (2) of dimensions in which the string can oscillate. However, we will now show that the high energy density of states of strings on a genus $h$ Riemann surface with cycles of order the string scale corresponds to $c_{e f f} \sim 2 h-2$. The point is that the fundamental group of the Riemann surface provides the required states, as follows.

Let us label the cycles of the Riemann surface in a symplectic basis by $a_{i}, b_{i}$ with $i=1, \ldots, h$, and their inverses by $a_{i}^{-1}, b_{i}^{-1}$ (keeping track of orientation). The fundamental group of a Riemann surface is given by "words" formed from paths going around these cycles, subject to the inverse relations $a_{i} a_{i}^{-1}=1=a_{i}^{-1} a_{i}$ and $b_{i} b_{i}^{-1}=1=b_{i}^{-1} b_{i}$ and the relation

$$
\prod_{i=1}^{h} a_{i} b_{i} a_{i}^{-1} b_{i}^{-1}=1
$$

Topologically stable string states arise for each independent element of this group. At large $h$, they correspond to a lattice random walk in a $2 h$-dimensional space: at each step, the string can wind in either direction around any of the $2 h-1$ available cycles (with the -1 here arising from the inverse relations precluding a step taken in the opposite direction from the previous step). Also at large $h$, the further relation (1.4) is a subleading effect. This identification of the high energy density of states with an isotropic $2 h$-dimensional random walk requires all the cycles of the same order, not exceeding the string scale in size. The selection rules determined by the fundamental group ensure that the states we are counting are classically stationary states before string interactions are taken into account.

At high energies, a typical high energy string state in $D-D_{c} \sim 2 h-2 \gg 1$ dimensions also behaves like a random walk in $D \sim 2 h$ dimensions [3]. Putting these two facts together, we learn that although at large radius a Riemann surface is clearly two-dimensional, if it is sufficiently isotropic as it approaches the string scale its high energy density of states behaves like a $2 h$-dimensional theory. 
This provides nontrivial evidence in favor of the conjecture that the Riemann surface theory can match at small radius onto a supercritical theory with matter central charge as large as

$$
c_{e f f}=D=2 h+\mathcal{O}(1)
$$

This large- $c_{\text {eff }}$ set of random walk string states supported by the fundamental group is the main observation of this note. In the next sections I will provide additional evidence for this crossover and its application to time dependent physics.

It is worth emphasizing that the value $2 h$ for $\hat{c}_{e f f}$ is not always attained as a Riemann surface shrinks; this depends on its moduli and spin structure. In particular, as a Riemann surface shrinks, it can undergo other transitions [4] [5] [6] which reduce its topology and/or separate it into distinct components if the spin structure is chosen appropriately and/or the surface approaches a factorization limit. Moreover, the cycles supporting the fundamental group elements might approach the string scale at different rates and at different times, yielding a smaller high energy density of states arising from the corresponding subgroup of the fundamental group. In any case the target space is supercritical.1

In this correspondence, dimensionality mutates into topologyl. In the remainder, we will elaborate on the physical context, interpretation, and perturbative self-consistency of this proposal. In the absence of additional ingredients, the Riemann surface at large radius evolves with time according to the vacuum Einstein equations. We will focus on this case in $\S 3$, where the supercriticality provides an intriguing approach to resolving the associated cosmological singularity and hence a useful role for the supercritical limits of perturbative string theory.

A more general setting in which to apply this connection is in a Riemann surface compactification with extra ingredients helping to stabilize it, as in [8]. This may slow or stop the time evolution in appropriate cases and permit comparison of metastable backgrounds of the Riemann surface and of the supercritical theory (itself with extra ingredients, perhaps along the lines of [9]). Other potential connections between critical and supercritical backgrounds of string theory were suggested in [10], and between critical and subcritical backgrounds in many works on closed string tachyon condensation.

1 Larger values of the UV central charge are also possible in sectors transverse to the Riemann surface; I will focus on the degrees of freedom that are identifiable on the Riemann surface side of the transition.

2 a feature suggested previously in $[7]$ in a different context 
These considerations apply also to any negatively curved space with a rich fundamental group, so it would also be interesting to apply this to hyperbolic 3-manifolds which can appear in models of $3+1$ dimensional cosmology.

\section{Renormalization Group Interlude}

In order to formulate perturbative string theory on a negatively curved target space, we must specify a well defined sigma model. The nonlinear sigma model on a negatively curved target space is strongly coupled in the ultraviolet, and requires a UV completion.3 Such a UV completion is easily obtained at the level of the two-dimensional matter sigma model by embedding the Riemann surface into a higher dimensional space swept out by some scalar fields $Y^{i}$. A potential $\mathcal{U}(Y)$ restricting the $Y$ fields to the Riemann surface is a relevant perturbation of the UV fixed point. In the UV, its effects go away, and the system lives on the space swept out by the $Y$ fields (or more generally an abstract CFT with a central charge $\hat{c}_{U V}>2$ ). In the IR, the potential produces a nonlinear sigma model on the Riemann surface.

Of course in a generic theory, the potential $\mathcal{U}(Y)$ may be strongly corrected upon flow to the IR, so that fixing the desired potential energy and the corresponding RG flow to the Riemann surface nonlinear sigma model would require significant fine tuning. However, in the case of worldsheet $(2,2)$ or $(0,2)$ supersymmetry, such corrections can be straightforwardly controlled. In particular, using the standard technology of gauged linear sigma models [11], it is possible to identify supercritical CFTs which provide the required UV fixed points in examples, as I review in the appendix. These examples include cases where a supercritical analogue of the type II GSO projection is available to the system, limiting the instabilities to which the system is susceptible as compared to more general analogues of the type 0 GSO projection.

As discussed in $\S 1$, the fundamental group of a Riemann surface of genus $h$ can support an effective central charge as high as $2 h$. However, the embedding spaces involved in the linear sigma model procedure for formulating the sigma model need not be of dimension $2 h$ (and need not be geometrical at the UV fixed point) though they all have UV central charge greater than $\hat{c}=2$. This corresponds to the fact discussed above that in order to

3 At the level of effective field theory of course one may alternatively define the sigma model with a cutoff. 
realize the maximal $2 h$ dimensional effective central charge supportable by the fundamental group, the surface must be rather symmetrical, and tachyonic modes need to be avoided, as the surface approaches the string scale. More generally, the supercritical starting point will realize a subset of this maximal $c_{e f f}$.

In the case of an embedding space with its own one-cycles, the Riemann surface can be embedded in such a way as to have some of its 1-cycles wrap 1-cycles in the embedding space. This provides a way to continue the winding gauge charges between the two descriptions in cases where they exist.

This linear sigma model definition of the sigma model on a Riemann surface does not in itself guarantee that time dependent backgrounds exist realizing an evolution between the supercritical and Riemann surface target spaces. However at large effective central charge the worlsheet matter RG provides in some cases a good approximation to the spacetime evolution, as we will review and apply in the next section. More generally, the RG problem may provide an indication of the transitions available in the full time dependent physics even in the case where the detailed evolution differs.

\section{Negative Curvature and Spacelike Singularities}

\section{1. $R G$ flow and time evolution at large $c_{e f f}$}

A higher genus Riemann surface, being negatively curved, is not by itself a static solution to string theory. At large radius, in the absence of extra stress energy sources its scale factor $a(t)$ evolves according to the vacuum Friedmann equation

$$
\left(\frac{\dot{a}}{a}\right)^{2}=\frac{1}{a^{2}}
$$

which forces the surface to expand or contract as a function of time according to

$$
a(t)= \pm\left(t-t_{0}\right)
$$

When $t$ reaches $t_{0}$, there is a spacelike singularity. Here for simplicity we consider constant spatial curvature; with more general choices the curvature perturbations smooth out at large $\left|t-t_{0}\right|$ but may be significant at small $\left|t-t_{0}\right|$.

A nonlinear sigma model on a Riemann surface target space flows toward large radius in the infrared. At large radius, the solution (3.1)(3.2) provides the correct dressing of this flow by worldsheet gravity to obtain a consistent background. As the Riemann surface 
shrinks as $t$ tends toward $t_{0}$ (either in the past or the future depending on the sign (3.2)), the effective central charge of the matter sigma model increases as discussed in the previous sections. This contrasts to the case of positively curved spatial slices, and the similar case of flat spatial slices with winding tachyon modes, where the singularity is associated with a lifting of degrees of freedom (a decrease in $c_{e f f}$ [4] [5] [6] (4)

It is difficult in general to follow the time evolution of this system through the string scale. However, the renormalization group flow in the matter sigma model becomes a good approximation to the time evolution in the case that the effective central charge is large. 0 One way to see this is as follows. Consider an RG flow from a UV fixed point to an expanding Riemann surface, such as that determined by the gauged linear sigma model in the previous section. The worldsheet action is semiclassically

$$
\mathcal{S}_{w s}=\mathcal{S}_{U V}+\int d^{2} \sigma \rho \mathcal{O} e^{\kappa X^{0}} \pm \int \mathcal{R}^{(2)} \sqrt{c_{e f f}} X^{0}
$$

where $\mathcal{O}$ is the relevant operator by which the UV CFT is perturbed at the beginning of the flow, and $\kappa$ is determined by the requirement that the operator $\mathcal{O} e^{\kappa X^{0}}$ be marginal. The scale transformations have a classical contribution arising from the worldsheet curvature term, and in the limit of large $c_{e f f}$, the two solutions for $\kappa$ scale like $\mp 1 / \sqrt{c_{e f f}}$ and $\pm \sqrt{c_{e f f}}$. To fix the signs, consider a dilaton evolving from strong to weak coupling. Then for fixed $\mathcal{O}$ dimension $\Delta$, the growing mode of the tachyon has $\kappa \propto 1 / \sqrt{c_{e f f}}$.

In the cases with $\kappa \propto \pm 1 / \sqrt{c_{e f f}}$, the contributions to the worldsheet path integral coming from fluctuations of $X^{0}$ itself are suppressed in this limit [13] [10]. The matter fluctuations contribute without any such suppression, and generate the RG flow of the theory, with the IR behavior corresponding to long times (large values of the $X^{0}$ zero mode $\left.X_{0}^{0}\right)$.

For this reason we will mostly focus on the case where the effective central charge is large as the system evolves from its supercritical description to a Riemann surface with string scale cycles, $V_{\Sigma} \sim(2 h-2) l_{s}^{2}$. As the surface further evolves to larger radius, its central charge drops closer to 2 , and the confluence of worldsheet RG and time evolution does not remain true. Hence the semiclassical approximation just employed to relate

4 A null singularity where the flow of degrees of freedom is as in the present case was suggested in 12$]$.

5 This point was first emphasized to me by J. Polchinski. I thank him and Xiao Liu for useful comments and suggestions on the evolution of the coupling. 
time evolution to RG flow does not suffice to describe the system all the way to the asymptotic future, but does provide a description of the transition region from the large$c_{e f f}$ supercritical theory to the large- $c_{e f f}$ string scale Riemann surface. As we will discuss in the next section, more general constructions are also possible, with transverse degrees of freedom which maintain semiclassicality for longer.

In this semiclassical regime, a small value of $\kappa$ through the supercritical phase and its transition to the string scale Riemann surface also serves to suppress particle production effects, which are computed similarly to [5] and are exponentially supressed in frequency/ $\kappa$. In order to trust these computations, the string coupling must be small; let us next address the behavior of the dilaton.

\subsection{Coupling}

Given a crossover to a supercritical phase, the large $c_{e f f}$ of the system sources the dilaton. An important question is the behavior of the dilaton during the crossover, and the direction of evolution of the string coupling in the supercritical phase.

The transition timescale is finite, of order $1 / \kappa$. The dilaton can be shifted

$$
\Phi \rightarrow \Phi-\Phi_{0}
$$

by a large constant $\Phi_{0}$, to obtain an arbitrarily weak coupling during the transitional epoch between the supercritical and Riemann surface phases.

However, to obtain a controlled description of the full background, one must address the behavior of the dilaton in the far past (fixing to the big bang case where the Riemann surface expands in the far future) in addition to addressing as in $\S 3.1$ the issue rate of time evolution in the background. Let us consider two cases in turn:

1) If we choose the signs suggested in $\S 3.1$, in which the tachyon describing the flow from the supercritical theory to the Riemann surface theory turns on slowly $\kappa \propto+1 / \sqrt{c_{e f f}}$, then the string coupling evolves toward weak string coupling as time evolves forward. This means that although the transition itself occurs during an epoch of weak coupling using (3.4), in the far past the system would be strongly coupled in the absence of other ingredients.

In the presence of other ingredients as in [9], we may shut off this strong coupling behavior by metastabilizing the dilaton. Moreover, one can include transverse degrees of freedom and a second tachyon field which grows toward the past and turns off by the 
time of the main supercritical $\leftrightarrow$ Riemann surface transition. This yields fewer degrees of freedom in the far past, slowing or stopping the growth of $g_{s}$ for $t \rightarrow-\infty$.

2) Conversely, we may choose the signs so that the dilaton is weakly coupled in the past (and evolves to a constant in the far future as $c_{e f f} \rightarrow c_{\text {critical }}$ ). Then a generic tachyon which effects the transition grows rapidly, yielding copious string production and precluding a simple identification of time evolution with RG flow. This evolution may still occur; the produced strings may simply dilute at late times as the Riemann surface expands, but the semiclassical methods outlined above do not yet apply to control the whole evolution (similarly to the situation with brane-antibrane annihilation). Again there is a modelbuilding opportunity to avoid this: in models where the relevant operator effecting the flow in the worldsheet matter theory is itself nearly marginal (with the dimension $\Delta$ of $\mathcal{O}$ in (3.3) very close to 2 ), this rapid time dependence would be avoided.

It would be interesting to build explicit models realizing one of these scenarios in which the string coupling is bounded throughout the background, and hence can be chosen everywhere small. In any case, given (3.4) the transition between the supercritical and Riemann surface regimes occurs at weak coupling.6

\section{Discussion}

All of this yields an interesting new time dependent process in string theory: a transition from high dimensionality to copious topology. The evidence for this crossover includes the striking ability of the Riemann surface fundamental group to support a large high energy density of states, and RG flow of linear sigma model realizations with its relation at large $c_{e f f}$ to the time dependent evolution. Clearly there is room for much more model building to explore this effect in detailed examples.

Although we focused here on the simplest negatively curved spaces, Riemann surfaces, the methods would generalize to hyperbolic 3-manifolds with large fundamental group. It raises an interesting possibility for resolving the spacelike singularity in negatively curved FRW solutions via a transition to a weakly coupled background of the supercritical theory. For this to occur, one must implement one of the more detailed scenarios in $\S 3$ to

6 A simpler example of a string-corrected spacelike singularity yielding self-consistent perturbative computations appeared in [5], where would-be divergences in the string path integral were shut off by a tachyon condensate. In the present case, the transition also involves a tachyon condensation (3.3), in this case connecting two backgrounds each with nontrivial central charge. 
simultaneously control the coupling and time dependence, not just in the Riemann surface case but in the case of hyperbolic 3-manifolds.7 The evolution between high and low effective dimensionality is a promising trend, as it opens up the possibility of a dynamical determination of the dimensionality.

This provides a new role for supercritical backgrounds, and renders it increasingly challenging to isolate the critical backgrounds of string theory; in the big bang case the process discussed here is perfectly consistent with the possibility of late time supersymmetry as the negatively curved spatial slices grow large. Conversely, late time supersymmetry, if present at all in nature, does not address very early cosmology which is likely to involve high scale supersymmetry breaking. Although it may at first sight seem more complicated to break supersymmetry, this is not always the case: supersymmetry-breaking effects can play a positive role in smoothing out singularities, as we have seen earlier in [4] [5] [6].

In the spacelike singularities studied in [5], and likely in similar cases with positively curved spatial slices, the singularity is replaced by a phase of tachyon condensate lifting closed string degrees of freedom, leading in the big bang case to an evolution as time moves forward from fewer to more degrees of freedom as measured by $c_{e f f}$. Similarly, as discussed in [14] [15], the evolution in realistic inflationary cosmology tends in this direction in terms of the evolution of the de Sitter entropy. The present set of examples works in the opposite way, with more degrees of freedom (as measured by $c_{e f f}$ ) appearing in the phase replacing the singularity. Both starting points are interesting to consider for the question of the space of initial (or final) conditions and its measure in light of the string landscape of late time solutions [9]. 8

Another intriguing aspect of the present study is the role of the simplifications that appear at large $c_{\text {eff }}$ : this aided our analysis of the high energy density of states and provided a relation between the RG and time dependent evolution. In other corners of M theory the simplifications arising in the presence of large numbers of degrees of freedom have played an important role. The limit of large dimensionality might play a simplifying

7 The nontrivial elements of $\pi_{1}$ may be well outside our horizon in realistic cosmological models of this sort, though it may be worth noting that the possibility of nontrivial topology is observationally accessible in the corner of parameter space where the cycles are just inside our Hubble volume.

8 Ultimately we may expect a prescription for summing over different spacetime backgrounds. Our Riemann surface topology is formulated via embedding in a space of higher dimensionality, which is reminiscent of worldsheet embeddings in spacetime. 
role in a way that is particularly suited to time dependent backgrounds and string-theoretic cosmology.9

Finally, the study of string target spaces at small radius has a rich tradition of connecting to mathematics. The case of broken supersymmetry is likely to continue this confluence, with techniques such as surgery [4] realized naturally in string theory, and generalizations of the CY-LG correspondence playing a role as discussed here. It would also be interesting if the present considerations could help clarify the physical context appropriate for interpreting [7].

\section{Acknowledgements}

I would like to thank A. Adams, S. Kachru, G. Horowitz, X. Liu, J. McGreevy, J. Polchinski, S. Shenker, and A. Tomasiello for very useful discussions, and S. Kachru, G. Horowitz, J. McGreevy, and J. Polchinski additionally for useful comments on the manuscript. I thank the KITP for hospitality during part of this work. This research is supported in part by the DOE under contract DE-AC03-76SF00515 and by the NSF under contract 9870115 .

\section{Appendix A.}

In this appendix, I explore two gauged linear sigma models realizing supercritical UV fixed points which flow upon relevant perturbation to nonlinear sigma models on Riemann surfaces.

Perhaps the simplest linear sigma model flowing to a Riemann surface nonlinear sigma model is obtained by realizing the surface via a degree $n$ algebraic defining equation in $\mathbb{C P}^{2}$. In the notation and conventions of [11], this is obtained in a $(2,2)$ gauged linear sigma model with the following field content and interactions. Three chiral multiplets $\Phi_{i}$ of charge 1 under a single $U(1)$ gauge symmetry alone would yield the $(2,2) \mathbb{C P}^{2}$ model. If we add another chiral multiplet $P$ with charge $-n$, the potential energy of the model is determined by the $D$-term and superpotential terms

$$
\begin{gathered}
D^{2}=\left(-n|P|^{2}+\sum_{i=1}^{3}\left|\Phi_{i}\right|^{2}-\rho\right)^{2} \\
W=P G_{n}(\Phi)
\end{gathered}
$$

9 Simplifications at large $D$ have also appeared in [9] 10] 10]. 
where $G_{n}$ is a degree $n$ polynomial in the $\Phi$ fields and $\rho$ is the Fayet-Iliopoulos term. The infrared physics is dominated by the space of field configurations minimizing the potential energy. At large positive $\rho$, the D-term and gauge invariance restrict the $\Phi$ 's to lie on $\mathbb{C I}^{2}$ (given a sufficiently generic polynomial $G_{n}$ which requires $P=0$ for any nonzero $\Phi_{i}$ ). The superpotential requires $G_{n}(\Phi)=0$, further restricting the fields to trace out a one complex dimensional locus.10 This surface is a two-torus $T^{2}$ if $n=3$.

The running of the coupling $\rho$ is determined in terms of the sum of the charges as

$$
\frac{d \rho}{d \ln (\mu)}=-n+3
$$

For $n=3, \rho$ is marginal. For $n>3, \rho$ runs to $+\infty$ in the IR and $-\infty$ in the UV, flowing toward large volume in the IR as expected for a negative curvature target space. The genus $h$ of the surface in the large radius geometrical phase is

$$
2-2 h=n(3-n)
$$

In this geometrical phase applicable in the deep IR, the effective central charge approaches $\hat{c}=2$ (where our "hat" notation is defined such that $\hat{c}=1$ corresponds to a single real dimension).

In the UV, $\rho \rightarrow-\infty$, and the model reduces to a Landau-Ginzburg orbifold. This model has central charge

$$
\hat{c}_{U V}=6 \times\left(1-\frac{2}{n}\right)>2
$$

Altogether, this model describes a flow starting from a UV CFT given by a LandauGinzburg orbifold. Perturbing by the relevant operator corresonding to $\rho$ yields a flow to a nonlinear sigma model on a Riemann surface. This provides a UV complete sigma model for the Riemann surface target space, which exhibits explicitly a decrease of the $c$ function.

As discussed in [4], this model is consistent with a chiral GSO projection if the sum of the charges $3-n$ is even, providing a non-anomalous $\mathbb{Z}_{2}$ subgroup of the chiral $R$ symmetry.

This model, while supercritical in the UV, does not realize the full $2 h$ dimensional target space suggested by the state counting in $\S 1$.11 Since its $c$ function is of order 1

10 For most values of $n$, there are also extra massive vacua as described in [11].

11 As discussed in $\S 1$, decay processes 洶 can occur at the string scale, depending on the moduli and spin structure. 
throughout, this model will also not provide a reliable indication of the time evolution of the corresponding spacetime string theory. To remedy this, we will next consider a slightly more complicated model which realizes a flow from a UV fixed point with a much higher UV central charge, $c \propto h$ for large $h$, to a Riemann surface of genus $h$.

Again in the notation and conventions of [1], consider the following $(2,2)$ symmetric gauged linear sigma model describing a complete intersection of hypersurfaces embedded in $\left(\mathbb{C P}^{2}\right)^{m}$. Start with a gauge group $U(1)^{m}$, and include for each factor in the gauge group 3 chiral multiplets of charge 1 (and uncharged under the remaining factors). That is, consider chiral multiplets $\Phi_{i}^{r}$ with $r=1, \ldots, m$ indexing the $U(1)$ factor under which $\Phi$ is charged, and with $i=1,2,3$ labeling the 3 fields of this charge. Add negatively charged chiral fields $P_{r}$ and $P_{r, r+1}$ as follows:

$$
\begin{aligned}
& P_{r} \quad r=1, \ldots, m \quad \text { charge }-3 \text { under } U(1)_{r} \\
& P_{r, r+1} \quad r=1, \ldots, m-1 \text { charge }-1 \text { under } U(1)_{r} \text { and } U(1)_{r+1}
\end{aligned}
$$

The corresponding $D$ terms of the model are of the form

$$
D_{r}^{2}=\left(-3\left|P_{r}\right|^{2}-\left|P_{r, r+1}\right|^{2}-\left|P_{r-1, r}\right|^{2}+\sum_{i=1}^{3}\left|\Phi_{i}\right|^{2}-\rho_{r}\right)^{2}
$$

for $r=2, \ldots, m-1$ (while for $r=1$ and $m$ one of the $\left|P_{r, r+1}\right|^{2}$ terms is absent). The sum of the charges being negative translates into the statement that the Fayet-Iliopoulos couplings $\rho_{r}$ run in the same direction as in our previous model: they run to large positive values $\rho_{r} \rightarrow+\infty$ in the IR, and $\rho_{r} \rightarrow-\infty$ in the UV.

At large positive $\rho$, the D-terms and gauge invariance restrict the $\Phi$ fields to lie on $\left(\mathbb{C P}^{2}\right)^{m}$. As in (A.2) above, superpotential terms and hence algebraic defining equations for our surface arise from gauge invariant terms of the form $P G(\Phi)$, with the charges of the $P$ fields (A.6) determining the degrees of the defining equations. The first set of $m$ equations corresponding to the $P_{r}$ (A.6) alone further restrict the $\Phi$ fields to live on $\left(T^{2}\right)^{m}$. Including the remaining set of $m-1$ equations yields enough independent equations to restrict the model to a one complex dimensional space. Because the Fayet-Iliopoulos couplings $\rho_{r}$ grow toward the IR, this surface grows in size in the IR and hence is a higher genus Riemann surface.

The adjunction formula [17] for the first Chern class again provides a simple way to compute the genus of this surface: it is given by

$$
\int c_{1}=2-2 h=6-6 m \Rightarrow h=3 m-2
$$


which reduces at large genus to $2 h \sim 6 \mathrm{~m}$.

In this model the UV theory arises for $\rho \rightarrow-\infty$. In the first model discussed above, this UV CFT was a Landau-Ginzburg model. In the present model, is a hybrid phase in the language of [11]. It has a UV central charge proportional to $h$, as can be seen as follows. Some $P$ fields in each D term (A.7) must be turned on. The zeroes of the potential then occur for $\Phi_{i}^{r}=0$ but for massless $\Phi$ fields their fluctuations about the minima yield contributions to the central charge as in Landau-Ginzburg theory. Consider for example the regime where $P_{r, r+1} \neq 0$. The corresponding superpotential terms mass up two of the three linear combinations of $\Phi^{r}$ fields for each $r$. If $P_{r}$ are also nonzero, the remaining $\Phi$ fields live on a Landau-Ginzburg theory with central charge $2 m / 3$. This is an underestimate for the UV central charge, as the $P$ fields can independently fluctuate to some extent. This hybrid phase is somewhat complicated, so it is worth noting that in heterotic versions of gauged linear sigma models, an LG phase rather than a hybrid phase can be obtained as in [18]. This occurs because in $(0,2)$ supersymmetry the defining equations arising in the $(0,2)$ superpotential terms need not involve extra scalar fields $P$ but instead multiply left-moving fermion superfields. Clearly there are many variants one can consider of this basic setup, and it would be interesting to look for simpler examples.

In this model, at large $2 h \sim 6 m$ the UV central charge is proportional to $h$. As such this RG flow not only defines the nonlinear sigma model on a genus $h$ surface, but does so in a way which comes close to realizing its full potential effective central charge (1.5). Because in particular it is large, this enables us to relate it to the main problem of interest, the time dependent evolution of spacetimes with negatively curved spatial components (in particular Riemann surfaces) as discussed in $\S 3$. 


\section{References}

[1] see e.g. D. Kutasov and N. Seiberg, "Number Of Degrees Of Freedom, Density Of States And Tachyons In String Theory And Cft," Nucl. Phys. B 358, 600 (1991).

[2] S. P. de Alwis, J. Polchinski and R. Schimmrigk, "Heterotic Strings With Tree Level Cosmological Constant," Phys. Lett. B 218, 449 (1989).

[3] see e.g. D. Mitchell and N. Turok, "Statistical Properties Of Cosmic Strings," Nucl. Phys. B 294, 1138 (1987).

[4] A. Adams, X. Liu, J. McGreevy, A. Saltman and E. Silverstein, "Things fall apart: Topology change from winding tachyons," arXiv:hep-th/0502021.

[5] J. McGreevy and E. Silverstein, "The tachyon at the end of the universe," JHEP 0508, 090 (2005) [arXiv:hep-th/0506130.

[6] G. T. Horowitz, "Tachyon condensation and black strings," JHEP 0508, 091 (2005) [arXiv:hep-th/0506166]; S. F. Ross, "Winding tachyons in asymptotically supersymmetric black strings," arXiv:hep-th/0509066.

[7] A. Iqbal, A. Neitzke and C. Vafa, "A mysterious duality," Adv. Theor. Math. Phys. 5, 769 (2002) [arXiv:hep-th/0111068].

[8] A. Saltman and E. Silverstein, "A new handle on de Sitter compactifications," arXiv:hep-th/0411271.

[9] R. Bousso and J. Polchinski, "Quantization of four-form fluxes and dynamical neutralization of the cosmological constant," JHEP 0006, 006 (2000) [arXiv:hep-th/0004134]; S. B. Giddings, S. Kachru and J. Polchinski, "Hierarchies from fluxes in string compactifications," Phys. Rev. D 66, 106006 (2002) [arXiv:hep-th/0105097]; E. Silverstein, "(A)dS backgrounds from asymmetric orientifolds," arXiv:hep-th/0106209; A. Maloney, E. Silverstein and A. Strominger, "De Sitter space in noncritical string theory," arXiv:hep-th/0205316; S. Kachru, R. Kallosh, A. Linde and S. P. Trivedi, Phys. Rev. D 68, 046005 (2003) [arXiv:hep-th/0301240]. et seq.

[10] S. Hellerman and X. Liu, "Dynamical dimension change in supercritical string theory," arXiv:hep-th/0409071.

[11] E. Witten, "Phases of $\mathrm{N}=2$ theories in two dimensions," Nucl. Phys. B 403, 159 (1993) [arXiv:hep-th/9301042].

[12] B. Craps, S. Sethi and E. P. Verlinde, "A matrix big bang," arXiv:hep-th/0506180.

[13] J. Polchinski, "A Two-Dimensional Model For Quantum Gravity," Nucl. Phys. B 324, 123 (1989); B. C. Da Cunha and E. J. Martinec, "Closed string tachyon condensation and worldsheet inflation," Phys. Rev. D 68, 063502 (2003) [arXiv:hepth/0303087]; E. J. Martinec, "The annular report on non-critical string theory," arXiv:hep-th/0305148.

[14] A. Strominger, "Inflation and the dS/CFT correspondence," JHEP 0111, 049 (2001) [arXiv:hep-th/0110087]. 
[15] M. Alishahiha, A. Karch and E. Silverstein, "Hologravity," JHEP 0506, 028 (2005) [arXiv:hep-th/0504056].

[16] A. Strominger, "The Inverse Dimensional Expansion In Quantum Gravity," Phys. Rev. D 24, 3082 (1981).

[17] see e.g. the explanation in T. Hubsch, "Calabi-Yau manifolds: A Bestiary for physicists,"

[18] J. Distler and S. Kachru, "(0,2) Landau-Ginzburg theory," Nucl. Phys. B 413, 213 (1994) [arXiv:hep-th/9309110]. 